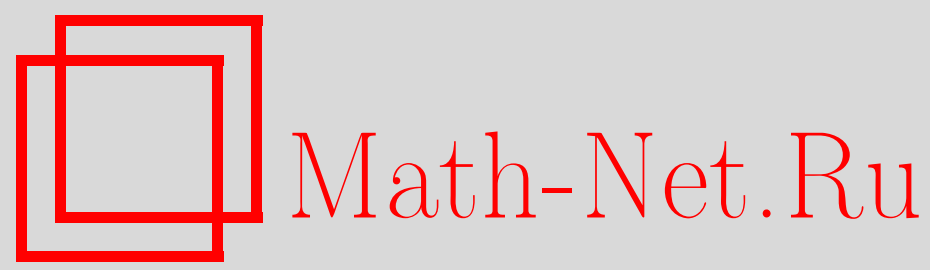

В. В. Горбацевич, Количественный аспект теоремы о стабилизации, Матем. заметки, 1998, том 64, выпуск 5, 788-791

DOI: https://doi.org/10.4213/mzm1455

Использование Общероссийского математического портала Math-Net.Ru подразумевает, что вы прочитали и согласны с пользовательским соглашением http://www.mathnet.ru/rus/agreement

Параметры загрузки:

IP: 54.166 .219 .16

26 апреля 2023 г., 18:14:49

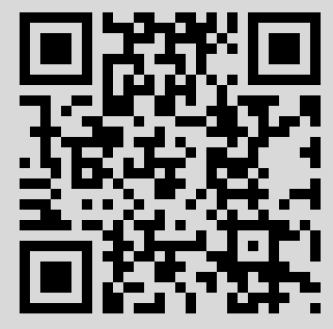




\section{КОЛИЧЕСТВЕННЫЙ АСПЕКТ ТЕОРЕМЫ О СТАБИЛИЗАЦИИ}

\section{В.В. Горбацевич}

Статья посвящена явному вычислению значений констант,входящих в теорему о стабилизации, доказанную автором в [1] (см. также [2]). Теорема о стабилизации сводит изучение вырождений конечномерных алгебр Ли (рассматриваемых с точностью до стабильного изоморфизма) к рассмотрению сжатий алгебр Ли некоторой, вообще говоря, большей размерности. Вкратце опишем используемые здесь понятия, отсылая за более подробным их изложением к работам [1], [2]. При этом мы будем рассматривать не только алгебры Ли, но и произвольные антикоммутативные конечномерные алгебры (как это было в [2]).

Пусть $A$-конечномерная алгебра на полем $K$, которое мы будем предполагать алгебраически замкнутым и имеющем характеристику 0 (обычно $K=\mathbb{C}$ ). Если в алгебре $A$ выбрать некоторьй базис, то эта алгебра определяется своим структурньм тензором $a_{i j}^{k}$, где $a_{i j}^{k} \in K$ - структурные константы. Через $\operatorname{Alg}_{n}(K)$ обозначим пространство всех структурных тензоров $n$-мерных алгебр над $K$. Через $\operatorname{Skew}_{n}(K)$ и $\operatorname{Lie}_{n}(K)$ обозначим подпространства в $\operatorname{Alg}_{n}(K)$, состоящие из антикоммутативных алгебр и алгебр Ли соответственно. На каждом из этих трех пространств естественным образом действует группа $\mathrm{GL}_{n}(K)$, орбиты которой состоят из всех изоморфных между собой алгебр (точнее, их структурных тензоров). Пространство $\operatorname{Alg}_{n}(K)$ и оба указанных его подпространства можно рассматривать как алгебраические подмножества в $K^{N}$, где $N=n^{3}$, поэтому на них естественным образом вводится топология Зариского. Далее, пространства $\mathrm{Alg}_{n}(K)$ при всевозможных $n \in \mathbb{N}$ образуют индуктивную систему (относительно естественных вложений $\operatorname{Alg}_{n}(K) \hookrightarrow \operatorname{Alg}_{n+1}(K)$, соответствующих переходу от алгебры $A$ к $\left.A \oplus K\right)$. Прямой предел этой системы обозначим через $\mathrm{Alg}_{\infty}(K)$. Аналогично вводятся пространства $\mathrm{Skew}_{n}(K)$ и $\mathrm{Lie}_{n}(K)$. Ha каждом из полученных трех пространств вводится топология,являющаяся прямым пределом топологий Зариского на пространствах из исходных систем. Две алгебры $A \in \operatorname{Alg}_{n}(K)$ и $B \in \operatorname{Alg}_{m}(K)$ назьваются стабильно изоморфным.ми, если они становятся изоморфными при прибавлении к ним некоторых конечномерных прямых слагаемых, имеющих тривиальное умножение (и потому изоморфных алгебрам вида $K \oplus K \oplus \cdots \oplus K$, где $K$ рассматривается не как поле, а как абелева алгебра Ли, т.е. алгебра с тривиальным умножением). Ясно, что пространство $\mathrm{Alg}_{n}(K)$ состоит из всех конечномерных алгебр над $K$, рассматриваемых с точностью до стабильного изоморфизма; так же можно описать и два других введенных выше предельных пространства. На этих пространствах имеется естественное действие группы $\mathrm{GL}_{\infty}(K)$ - прямого предела групп $\mathrm{GL}_{n}(K)$ (образующих индуктивную систему относительно очевидным образом определенных вложений), причем если на этой группе ввести топологию прямого предела топологий Зариского, то указанное действие будет непрерывно. Для алгебры $A \in \operatorname{Alg}_{n}(K)$ мы через $O_{n}(A)$ обозначим ее орбиту относительно действия группы $\mathrm{GL}_{n}(K)$. Орбита любой алгебры с нетривиальным умножением не будет замкнутой; любую алгебру, лежащую в замыкании этой орбиты, называют сжсатием исходной алгебры. Алгебру $A$ можно рассматривать и как лежаную в $\operatorname{Alg}_{\infty}(K)$; через $O_{\infty}(A)$ обозначим ее орбиту относительно действия группы $\mathrm{GL}_{\infty}(K)$. Алгебры, лежащие в замыкании этой орбиты, назьваются выро ждениями исходной алгебры $A$. Ясно, что любое сжатие алгебры $A$ можно рассматривать и как ее вырождение, обратное же, вообще говоря, неверно. Например, для двумерной неабелевой разрешимой алгебры Ли $R_{2}$ (такая алгебра над данным полем $K$ единственна с точностью до изоморфизма) имеет вырождение в трехмерную нильпотентную неабелеву алгебру Ли $N_{3}$ (см., например, [1]), это вырождение соответствует сжатию алгебры Ли $R_{2} \oplus K$ в $N_{3}$, а сжатия любой алгебры в алгебру большей размерности невозможны просто по определению. Можно было бы предполагать, что, прибавляя к исходной алгебре $A$ прямые слагаемые с тривиальным умножением все большей размерности (что не меняет эту алгебру как элемент пространства $\mathrm{Alg} \infty(K)$ ), мы будем получать все более и более сложные замыкания орбит и тем самым все новые и новые вы-

Работавыполнена при финансовой поддержке Международного научного фонда, грант RO 4300, и Российского фонда фундаментальных исследований, грант № 95-101263. 
рождения этой алгебры $A$. Однако, как было доказано в [1], начиная с некоторого момента в этом процессе наступает стабилизация. А именно, имеет место следующая теорема о стабилизации.

Теорема 1 [1]. Пусть $A \in \operatorname{Alg}_{\infty}(K)$ некоторая $n$-мерная алгебра. Тогда если имеется ее вырохдение в некоторую алгебру $B \in \operatorname{Alg}_{\infty}(K)$, то существует сжатие алгебры $A \oplus K^{n}$ в некоторую алгебру $B^{\prime}$, стабильно изоморфную $A$.

Другими словами, для $n$-мерной алгебры $A$ любое сжатие алгебры вида $A \oplus K^{m}$ при $m \geqslant n$ "пропускается" через некоторое сжатие алгебры $A \oplus K^{n}$ в алгебру $B^{\prime}$, стабильно изоморфную алгебре $B$. Эта теорема дает возможность описьвать замыкания орбит в $\operatorname{Alg}_{\infty}(K)$. Для этого в [1], [2] было введено и изучено понятие уровня алгебр (который характеризует сложность операции умножения в алгебре) и классифицированы антикоммутативные алгебры первых трех уровней. Данная заметка посвящена количественному уточнению формулировки теоремы о стабилизации, что позволит применять ее более эффективно.

Обозначим через $p_{n}$ наименьшее из таких натуральных чисел $p$, что для любой алгебры $A$ размерности $\leqslant n$ любое сжатие алгебры $A \oplus K^{m}$ при произвольном $m \in \mathbb{N}$ пропускается через некоторое сжатие алгебры $A \oplus K^{p}$ (рассматриваемое с точностью до стабильного изоморфизма). В силу теоремы 1 имеем $p_{n} \leqslant n$. Следующее утверждение дает точное выражение $p_{n}$ через $n$ в случае, если рассматриваются только алгебры Ли. Соответствующее число $p_{n}$ мы обозначим $p_{n}^{\text {Lie }}$. Из теоремы 1 следует, что $p_{n}^{\mathrm{Lie}} \leqslant n$.

Теорема 2. Справедливы соотношения

$$
p_{n}^{\text {Lie }}= \begin{cases}n-1 & \text { npu } n=1,2,4 \\ n & \text { npu } n=0,3 \text { u } n \geqslant 5 .\end{cases}
$$

ДокАЗАТЕльство. Пусть $A$ - некоторая $n$-мерная алгебра Ли над полем $K$. Если $n \leqslant 1$, то $A$ - нулевая алгебра Ли $\{0\}$ или одномерная (которая изоморфна $K$ ). Очевидно, что тут $p_{n}=0$. Если $n=2$, то $A$ изоморфна $K^{2}$ или алгебре Ли $R_{2}$, описанной выше. В [2] доказано, что алгебра Ли $R_{2} \oplus K^{m}$ может быть сжата только в алгебры Ли вида $A^{\prime} \oplus K^{m^{\prime}}$, где алгебра Ли $A^{\prime}$ изоморфна $\{0\}, R_{2}$ или $N_{3}$, a $m^{\prime}=m+2-\operatorname{dim} A^{\prime}$. Отсюда видно,что любое сжатие алгебры Ли $R_{2} \oplus K^{m}$ пропускается через сжатие алгебры Ли $R_{2} \oplus K$, а потому $p_{2}^{\text {Lie }} \leqslant 1$. Так как имеется сжатие алгебры Ли $R_{2} \oplus K$ в $N_{3}$, получаем, что $p_{1}^{\text {Lie }}=1$.

Пусть теперь $n \geqslant 3$. Предположим, что алгебра Ли $A$ такова, что $[A, A]=A$. В [2] для произвольной антикоммутативной конечномерной алгебры Ли $A$ построена такая 2 -нильпотентная (т.е. класс нильпотентности которой равен двум) алгебра Ли $m(A)$, в которую может быть сжата алгебра Ли $A \oplus K^{n}$. Из условия $[A, A]=A$ следует, что эта алгебра Ли $m(A)$ будет несократима, т.е. она не изоморфна никакой алгебре Ли вида $B \oplus K^{l}$ при $l>0$. Из этого следует, что $p_{n}^{\text {Lie }} \geqslant n$. В силу теоремы 1 имеем $p_{n}^{\text {Lie }} \leqslant n$, поэтому $p_{n}^{\text {Lie }}=n$. Но это равенство мы получили только для тех значений $n$, для которых сушествует $n$-мерная алгебра Ли с условием $[A, A]=A$. Нетрудно показать, что это имеет место только при $n=3$ и $n \geqslant 5$. А именно, положим $A_{n}=\operatorname{sl}_{2}(K)+\varphi K^{n-3}$, где $\varphi=\varphi_{n-3}: \mathrm{sl}_{2}(K) \rightarrow \mathrm{gl}_{n-3}(K)$ - неприводимое представление трехмерной простой алгебры Ли $\operatorname{sl}_{2}(K)$. Легко проверить,что при $n \neq 4$ имеем $\left[A_{n}, A_{n}\right]=A_{n}$. Поэтому при $n=3$ и $n \geqslant 5$ приходим к равенству $p_{n}^{\text {Lie }}=n$. Остается рассмотреть случай $n=4$. Имеем $p_{4} \leqslant 4$. Покажем вначале, что $p_{4} \geqslant 3$. Для этого рассмотрим алгебру Ли вида $A=K+\varphi K^{3}$, где $\varphi$ - линейное отображение такое, что $\varphi(1)=\operatorname{diag}\left(\lambda_{1}, \lambda_{2}, \lambda_{3}\right)$ и числа $\lambda_{i} \in K$ попарно различны. Из результатов работы [2] следует, что существует сжатие алгебры Ли $A \oplus K^{3}$ в алгебру Ли вида $B=K+{ }_{\psi} K^{6}$, где $\psi(1)$ - нильпотентная матрица шестого порядка, равная прямой сумме трех жордановых клеток $\left(\begin{array}{ll}0 & 1 \\ 0 & 0\end{array}\right)$. Ясно, что эта алгебра Ли $B$ несократима, а потому должно быть $p_{4}^{\text {Lie }} \geqslant 3$. Докажем теперь, что $p_{4}^{\text {Lie }} \leqslant 3$. Для этого заметим, что четырехмерная алгебра Ли $A$ над алгебраически замкнутым полем характеристики 0 или разрешима, или изоморфна алгебре Ли вида $\mathrm{sl}_{2}(K) \oplus K$. Как в том, так и в другом случае $\operatorname{dim}[A, A] \leqslant 3$. Если при этом еще справедливо неравенство $\operatorname{dim}[A, A] \geqslant \operatorname{dim} Z(A)$, где $Z(A)$ обозначает центр алгебры Ли $A$, то из теоремы 3 (см. далее) следует, что для таких алгебр Ли $p_{4} \leqslant 3$. Если же алгебра Ли $A$ такова, что $\operatorname{dim}[A, A]<\operatorname{dim} Z(A)$, 
то обязательно $\operatorname{dim} Z(A) \leqslant 2$. Если $\operatorname{dim}[A, A]=0$ или 1 , то $A$ будет изоморфна $K^{4}$ или $R_{2} \oplus K^{2}$, а для этих алгебр, очевидно, можно взять $p=0$. Если же $\operatorname{dim}[A, A]=2$, то $\operatorname{dim} Z(A) \geqslant 3$, откуда сразу следует, что $A$ должна быть абелевой (что невозможно в данном случае). Из сказанного следует, что при $n=4$ во всех возможных случаях можно брать $p=3$. Но тогда в сочетании с $p_{4} \leqslant 4$ это приводит к равенству $p_{4}^{\text {Lie }}=3$.

Отметим, что кроме чисел $p_{n}^{\text {Lie }}$ можно рассматривать аналогичные числа применительно к пространству $\operatorname{Alg}_{\infty}(K)$ всех конечномерных алгебр и к пространству Skew $\infty(K)$ всех конечномерных антикоммутативных алгебр над $K$. При этом если $n=3$ или $n \geqslant 5$, то $p_{n}=n$ и для этих двух пространств, ибо это максимально возможные (в силу теоремы 1 ) значения для $p_{n}$, а они достигаются уже для алгебр из пространства $\operatorname{Lie}_{\infty}(K)$, содержащегося в каждом из двух других пространств. Что касается значений $p_{n}$ при $n=1,2,4$, то для $A \lg _{\infty}(K)$ они равны $1,2,4$ соответственно (и потому $p_{n}=n$ при всех значениях $n$ ), а для Skew $\infty(K)$ эти значения равны $0,1,4$ соответственно. Покажем это. Рассмотрим вначале пространство $\operatorname{Alg}_{\infty}(K)$. Через $A_{1}$ обозначим такую одномерную алгебру, которая для некоторого своего образующего элемента $e_{1}$ удовлетворяет соотношению $e_{1}^{2}=e_{1}$. Положим $A_{n}=A_{1} \oplus A_{1} \oplus \cdots \oplus A_{1}$ (всего $n$ слагаемых). Ясно, что $A_{n}^{2}=A_{n}$, поэтому из рассуждения, приведенного при доказательстве теоремы 2, следует, что при любом значении числа $n \geqslant 1$ (ибо указанные вьшше алгебры имеются при любом таком $n$ ) вьполнено равенство $p_{n}=n$. Что касается пространства $\operatorname{Skew}_{\infty}(K)$, то равенство $p_{1}=0$ для него следует из того, что одномерная антикоммутативная алгебра обязательно абелева (в частности, она будет лиевой). Далее, легко проверить, что и любая двумерная антикоммутативная алгебра является лиевой. Поэтому значения $p_{n}$ при $n=1,2$ для пространств $\operatorname{Skew}_{\infty}(K)$ и $\operatorname{Lie}_{\infty}(K)$ будут одинаковы. Что же касается размерности 4 , тут рассмотрим такую четырехмерную антикоммутативную алгебру $C_{4}$, которая в некотором базисе $e_{1}, e_{2}, e_{3}, e_{4}$ задается соотношениями $e_{i} e_{j}=e_{i+j}$ при $i>j$ (остальные соотношения либо следуют из антикоммутативности или же являются нулевыми), причем сумма $i+j$ берется по модулю 4 . Ясно, что для так построенной алгебры имеем $C_{4}^{2}=C_{4}$, a потому снова из рассуждения, приведенного при доказательстве теоремы 2 , получаем, что $p_{4}=4$ для пространства Skew $\infty(K)$. Тем самьм, значения $p_{n}$ вычислены для всех трех пространств, хотя наиболее интересным для нас является случай пространства конечномерных алгебр Ли.

Значения $p_{n}$, рассмотренные выше, относились ко всем конечномерным алгебрам рассматриваемого класса. Теперь мы введем более индивидуализированные величины. А именно, для произвольной конечномерной алгебры $A$ над полем $K$ рассмотрим число $p(A)$, равное наименьшему из таких чисел $p \in \mathbb{N}$, для которых произвольное сжатие алгебры вида $A \oplus K^{m}$ при любом $m$ может быть пропущено через сжатие алгебры $A \oplus K^{p}$. Из теоремы 1 следует, что всегда $p(A) \leqslant n$. Далее, в [1, теорема 3$]$ было фактически доказано (хотя там рассмотрены только антикоммутативные алгебры и не используется введенное в данной статье обозначение $p(A))$, что если алгебра $A$ такова, что $A^{2}=Z(A)$, то $p(A)=0$ (здесь $Z(A)=\{X \in A \mid X Y=Y X=0$ для всех $Y \in A\}-$ центр алгебры $A$ ). Из этого утверждения в [1] было выведено, что если $A$ - произвольная несократимая 2-нильпотентная алгебра Ли, то для нее тоже $p(A)=0$. Эти результаты позволяют доказать следующее общее утверждение.

Теорема 3. Пусть $A$ - произвольная антикоммутативная конечномерная алгебра над полем $K$. Тогда если $\operatorname{dim} A^{2} \geqslant \operatorname{dim} Z(A)$ (это условие эквивалентно несократимости алгебры $A)$, то $p(A)=\operatorname{dim} A^{2}-\operatorname{dim} Z(A)$.

ДокАЗАтЕльство. Положим $l=\operatorname{dim} A^{2}-\operatorname{dim} Z(A)$ и рассмотрим алгебру $A_{1}=A \oplus K^{l}$. Для полученной алгебры $A_{1}$ имеем, очевидно, равенство $\operatorname{dim} A_{1}^{2}=\operatorname{dim} Z\left(A_{1}\right)$. Отсюда в силу сформулированного результатаиз [1] имеем $p\left(A_{1}\right)=0$. Но это равенство означает, что $p(A) \leqslant \operatorname{dim} A^{2}-$ $\operatorname{dim} Z(A)$. Для доказательства обратного неравенства рассмотрим 2-нильпотентную алгебру $m(A)$, построенную в [2], и ее редукцию, т.е. разложение в прямую сумму вида $m(A)=B \oplus K^{t}$, где алгебра $B$ несократима. Из [2, предложение 1] следует, что $t=\operatorname{dim} A / A^{2}+\operatorname{dim} Z(A)$. Но тогда ясно, что $p(A) \geqslant \operatorname{dim} A-t=\operatorname{dim} A^{2}-\operatorname{dim} Z(A)$. В сочетании с неравенством, доказанным выше, это приводит к равенству $p(A)=\operatorname{dim} A^{2}-\operatorname{dim} Z(A)$.

Если $A$-несократимая 2-нильпотентная алгебра Ли, то для нее $\operatorname{dim}[A, A]=\operatorname{dim} Z(A) ;$ поэтому в силу теоремы 3 имеем $p(A)=0$, т.е. приходим к указанному выше результату из [1]. В дополнение 
к теореме 3 укажем, что для произвольной антикоммутативной алгебры $A$ всегда имеет место неравенство $p(A) \geqslant \operatorname{dim} A^{2}-\operatorname{dim} Z(A)$. Дело в том, что при $\operatorname{dim} A^{2}<\operatorname{dim} Z(A)$ оно тривиальным образом выполнено, а при $\operatorname{dim} A^{2} \geqslant \operatorname{dim} Z(A)$ вытекает из утверждения теоремы 3.

Пусть $A$ - некоторая конечномерная антикоммутативная алгебра над $K$. Вообще говоря, алгебра $A$ сократима. В силу ее конечномерности существует такое разложение в прямую сумму $A=A_{1} \oplus K^{m}$ при некотором $m \in \mathbb{N}$, что алгебра $F_{1}$ уже будет несократимой. Алгебра $A_{1}$ назьвается редукиией алгебры $A$, она определяется алгеброй $A$ однозначно с точностью до изоморфизма (см. [2]). Следующее утверждение непосредственно вытекает из теоремы 3.

СледствиЕ 1. Пусть $A$ - некоторая конечномерная антикоммутативная алгебра, а $A_{1}$ - ее редукиия. Тогда $p(A)=\operatorname{dim}\left(A_{1}\right)^{2}-\operatorname{dim} Z\left(A_{1}\right)$.

Алгебру $A$ будем назьвать монолитной, если $A^{2}=A$ и $Z(A)=\{0\}$. Например, монолитной будет любая полупростая алгебра Ли. Для алгебр Ли вместо $A^{2}$ обычно пишется $[A, A]$. Разрешимая алгебра Ли не может быть монолитной, а если монолитна некоторая неразрешимая алгебра Ли,то ее радикал должен быть нильпотентньм. Монолитность алгебры Ли означает, что попытки построить для нее ряд коммутантов или центральный ряд (верхний или нижний) приведут к тривиальному результату.

СледствиЕ 2. Пусть $A$ - монолитная конечномерная алгебра. Тогда $p(A)=0$.

\section{СПИСОК ЦИТИРОВАННОЙ ЛИТЕРАТУРЫ}

1. Горбацевич В. В. // Изв. вузов. Матем. 1992. №2. С. 24-32. 2. Горбацевич В. В. // Алгебра и анализ. 1993. Т. 5. № 3. С. 100-118.

Московский авиационно-технологический институт им. К. Э. Циолковского

E-mail: vladimir@edunet.ru 